\title{
Chiral fluids: a few theoretical issues
}

\author{
V. I. Zakharov ${ }^{1,2, a}$ and O. V. Teryaev ${ }^{3,4}$ \\ ${ }^{1}$ NRC Kurchatov Institute - ITEP, 117218 Moscow, Russia, \\ ${ }^{2}$ School of Biomedicine, Far Eastern Federal University, 690950 Vladivostok, Russia, \\ ${ }^{3}$ JINR \\ ${ }^{4}$ Moscow Institute of Engineering
}

\begin{abstract}
We review briefly a few topic concerning physics of fluids whose constituents are massless fermions interacting in chiral invariant way. Macroscopic manifestations of the chral anomaly is one of central issues. Another topic is ultraviolet vs infrared sensistivity of chiral magnetic and vortical effects. To clarify dynamical issues involved we rely mostly on a (well-known) toy model of pionic superfluidity.
\end{abstract}

\section{Introduction}

By chiral fluids one understands fluids whose constituents are massless fermions. In presence of external electric and magnetic fields there arises the chiral anomaly, as usual. Manifestations of the chiral anomaly in the standard set up of field theory have been exhaustively studied. If instead of the vacuum-to vacuum matrix elements one turns to the matrix elements over medium the expectation would be-we guess-that the effect of the anomaly is damped down. Indeed, the anomaly is a quantum effect and is sensitive to phases of amplitudes. Interaction with medium would lead to the loss of coherence and wash out the effect of the anomaly. Superfluidity is an exceptional and well understood case when quantum effects survive macroscopically.

On the background of such intuitive picture the results of an explicit evaluation of the effect of the anomaly in the hydrodynamic approximation [1] turned unexpected. Namely, It was found that the effect of anomaly survives and leads to macroscopic, so called chiral effects. We mention here two such effects, or novel contributions to the vector and axial-vector currents $j_{\alpha}$ and $j_{\alpha}^{5}$, respectively:

$$
\begin{gathered}
j_{\alpha}=n_{V} u_{\alpha}+C_{\text {anom }} e^{2} \mu_{A} B_{\alpha}, \\
j_{\alpha}^{5}=n_{A} u_{\alpha}+C_{\text {anom }}\left(\mu_{V}^{2}+\mu_{A}^{2}\right) \omega_{\alpha},
\end{gathered}
$$

where $u_{\alpha}$ is the 4-velocity of an element of the fluid, $n_{V}, n_{A}$ are the sum and difference of the densities of the right- and left-handed constituents, $\mu_{V}, \mu_{A}$ are the corresponding chemical potentials, $B_{\alpha}$ is the electromagnetic field in the medium, $B_{\alpha}=(1 / 2) \epsilon_{\alpha \beta \gamma \delta} u^{\beta} F^{\gamma \delta}$ and $\omega_{\alpha}$ is the vorticity, $\omega_{\alpha}=(1 / 2) \epsilon_{\alpha \beta \gamma \delta} u^{\beta} \partial^{\gamma} u^{\delta}, e$ is the electromagnetic coupling. Finally, $C_{a n o m}$ is a constant which appears in front of the anomaly and its presence signifies the fact that the corresponding term would be absent

ae-mail: vzakharov@itep.ru 
in absence of the anomaly. The value of the constant $C_{\text {anoma }}$ depends on the number of massless constituents and $C_{\text {anom }}=1 /\left(2 \pi^{2}\right)$ in case of a single Dirac particle of charge $e$. All the chiral effects are proportional to $C_{\text {anom }}$.

This amusing conclusion that the effect of the anomaly survives in the hydrodynamic limit is not the only feature which triggerred a lot of interest in the chiral effect. The first years of the development were summarized in a volume of reviews [2]. Here, we will pick up only two points.

Turn first to chiral magnetic effect, see the term proportional to $C_{a n o m}$ in the first line of the expression (1). At least at its face value, the chiral magnetic effect is a flow of electric current along an external magnetic field. Then one can argue [3] that this current is dissipation-free (in a CP-invariant theory). The proof is straightforward and is based on the observation that the chiral magnetic current and the standard electric current have opposite parities under reflection of time. Thus, the chiral magnetic effect seems to echo superfluidity. The puzzle is that there is no microscopic mechanism known which would result in dissipation-free transport in case of the chiral magnetic current.

The other observation in point is that the currents (1) unify helical motions of macroscopic and microscopic degrees of freedom. Indeed, concentrate on the zeroth component of the axial current (1)

$$
j_{0}^{5}=\left(n_{R}-n_{L}\right) u_{0}+C_{\text {anoma }}\left(\mu^{2}+\mu_{5}^{2}\right) \vec{\Omega} \cdot \vec{v},
$$

The first term in the right side is the difference of densities of right- and left-handed constituents. While the other term is non-vanishing for a macroscopic helical motion. Furthermore, one can argue $[4,5]$ that unification of these terms eventually results in possiblity of transition between the two terms (so that the total charge is conserved). There arises a similar mixing between helicity of macroscopic magnetic fields and difference in number of right- and left-handed photons. In fact, it is the instability induced by the mixing of the two electromagnetic terms which was studied in most detail [6-8].

Again, the mechanism of the transition between the microscopic and macroscopic motions is difficult to visualize. Indeed, chirality of the massless constituents is defined without any reference to externally determined directions. A particle remains, say, left-handed while moving in various directions. On the other hand, the macroscopic helical motion is triggerred by external forces. The macroscopic motion can be imitated by microscopic degres of freedom only if the massless particles start moving predominantly in a particular direction. To our knowledge, there is no mechanism to produce such an alignment of the momenta of the constituents.

In conclusion of this brief introduction let us emphasize that the literature on the chiral fluids is huge and in no way is referred to here systematically. We cite papers mostly in cases when we reproduce directly argumentation of these papers. Otherwise, the reader is referred to the corresponding original papers as well as review papers for more comprehensive lists of references.

\section{Extra conservation laws in case of ideal fluids}

Equations (1) have been reproduced many times, by different techniques. The standard derivation of (1) starts with external elctromagnetic fields present. In the final answer one is free to put fileds to zero. Note, however, that the expression (1) for the axial current contains the chiral vortical effect also in absence of external fields. Therefore, we come to a paradoxical conclusion that appearance of $C_{\text {anom }}$ in front of a particular term in the current is not actually a proof of its relation to the anomaly!

An alternative derivation of the anomalous terms which is valid also in absence of the external fields was suggested in Ref. [9]. The point is that in thermodynamics one introduces effective Hamiltonian $\hat{H}_{e f f}$ which is related to the original Hamiltonian of the system $\hat{H}_{0}$ as:

$$
\hat{H}_{e f f}=\hat{H}_{0}-\mu \cdot \hat{Q},
$$


where the chemical potential $\mu$ is conjugated to the charge $Q$. In the hydrodynamic set up, the extension (3) corresponds to introduction of effective Lagrangian:

$$
\delta L_{e f f}=\mu u_{\alpha} j^{\alpha}
$$

where $\int d^{3} x j^{0} \equiv Q$.

Imagine now that we start calculating matrix elements of the currents in the hydrodynamic approximation beginning with the fundamental Lagrangian modified according to (4). Applying then the Adler-Bardeen theorem we would uniquely fix an anomalous contribution which can be generated from the standard anomaly by the substitution [10]

$$
e A_{\alpha} \rightarrow e A_{\alpha}+\mu u_{\alpha}
$$

One can readily check that in this way one reproduces the standard chiral effects.

This completes the derivation of the chiral vortical effect in absence of external fields. Solving one problem, however, brings us to confront another problem. Namely, if one evaluates directly Feynman graphs the bare triangle graph is singled out by nonconservation of the axial current. In case of the chiral vortical effect:

$$
\left(\partial^{\alpha} j_{\alpha}^{5}\right)_{\text {vortical }} \sim \vec{\Omega} \cdot \vec{a},
$$

$\Omega_{i}=\frac{1}{2} \epsilon_{i j k} \partial^{j} v^{k}, a_{i}=d v_{i} / d t$ and we use non-relativistic approximation for simplicity (c.f. [11]). However, it is well known that introduction of a chemical potential or temperature does not affect the chiral anomaly. Indeed, the anomaly can be derived entirely in terms of UV regularization which is not sensitive to introduction of a medium.

A way to resolve this paradox is to realize that the r.h.s. of Eq. (6) does not vanish on the equations of motion which follow from the fundamental Lagrangian. But the accelaration $\vec{a}=0$ for the fluid in equilibrium, see, in particular $[12,13]$. Thus, the matrix element (6) vanishes in the equilibrium, or on the equations of motion for the medium. Note that in the limit of ideal fluid the velocities $\vec{v}$ do not vanish, generally speaking, and the fluid can have a nontrivial, conserved helical charge, see Eq. (2). Introduction of dissipation would generically lead finally to $\vec{v}=0$. Thus, the helical charge is not conserved for viscous fluid. This observation is not in contradiction with our derivation of the chiral vortical effect in terms of the Feynman graphs. Indeed, unitary field theory cannot incorporate dissipation.

\section{Ideal fluid in external gravitational field}

\subsection{Conservation laws}

In this section we consider motion of chiral fluid in an external gravtational field. One of purposes of introduction of the gravitational field is to address theory of thermal chiral effects. Indeed, according to Luttinger [14] external gravitational field imitates the effect of a nonvanishing gradient of temperature. Therefore, studying chiral fluids in external gravitational filed can be useful to appreciate chiral thermal effects, see [15] and references therein.

Let us concentrate on conservation laws for the axial current, see (1). As we argued in the preceding section, in case of ideal fluid (and absence of external fields) there are actually two separately conserved currents:

$$
\begin{array}{r}
\partial_{\alpha}\left(n_{A} u^{\alpha}\right)=0, \\
\epsilon^{\alpha \beta \gamma \delta} \partial_{\alpha}\left(\mu u_{\beta}\right) \partial_{\gamma}\left(\mu u_{\delta}\right)=0 .
\end{array}
$$


The first line here is the conservation of the standard hydrodynamic current which is granted since we do not include external electromagnetic fields. The second line is then consequence of (1). The physical meaning of the latter conservation law is the vanishing acceleration, $\vec{a}=0$, see for the discussion above.

As the next step, switch on an external gravitational field. The ordinary derivatives $\partial_{\alpha}$ are replaced then by covariant ones, $D_{\alpha}$ and for any current $j_{\alpha}$ conserved in the flat-space limit one gets:

$$
\partial_{\alpha} j^{\alpha}=0 \rightarrow D_{\alpha} \mathcal{J}^{\alpha}=0,
$$

where the current $\mathcal{J}_{\alpha}$ transforms as a vector under general coordinate transformations. In the flatspace limit the current $\mathcal{J}_{\alpha}$ coincides with $j_{\alpha}$. In curved space, there are deviations of $\mathcal{J}_{\alpha}$ from $j_{\alpha}$ which are fixed by the transformation properties of $\mathcal{J}_{\alpha}$ under coordinate change.

In presence of gravitational field the conservation law now looks as:

$$
\mathcal{J}_{; \mu}^{\mu}=\frac{\partial \mathcal{J}^{\mu}}{\partial x^{\mu}}+\Gamma_{\mu \rho}^{\mu} \mathcal{J}^{\rho}=0,
$$

where $\mathcal{J}_{; \mu}^{\mu} \equiv D_{\mu} \mathcal{J}^{\mu}$ and $\Gamma_{\nu \rho}^{\mu}$ are Christoffel symbols. We will be interested in the lowest-order effect of gravity and assume that deviations from the Minkowskian space are weak: $g_{\mu \nu}=\delta_{\mu \nu}+h_{\mu \nu},\left|h_{\mu \nu}\right| \ll 1$. In case of a weak field the general expression (9) becomes:

$$
\partial_{\mu} \mathcal{J}^{\mu} \approx-(1 / 2) \mathcal{J}^{\mu} \partial_{\mu} h
$$

where $h \equiv h_{00}-\Sigma_{i} h_{i i}$. The $h_{00}$ component, as usual, is fixed by the equivalence principle:

$$
g_{00}-1 \approx 2 \phi_{g r} \approx-2 \vec{a} \cdot \vec{x}
$$

where $\vec{a}$ is the acceleration.

Applying these general relations to our case (7) we get to first order in gravtational field:

$$
\begin{array}{r}
\partial_{\alpha}\left(n_{A} u^{\alpha}\right)=0, \\
\partial_{\alpha} \epsilon^{\alpha \beta \gamma \delta}\left(\mu u^{\beta}\right) \partial^{\gamma}\left(\mu u^{\delta}\right)=(\text { const }) \mu^{2} \vec{\Omega} \cdot \vec{a}
\end{array}
$$

Note absence of corrections to the standard hydrodynamic current in the ideal-fluid limit. It is a consequence of the relation $u^{\alpha} a_{\alpha}=0$ where $a_{\alpha}=(d / d s) u_{\alpha}$. Indeed, if the fluid is at rest, then $(d / d t)\left(n u_{0}\right)=0$ according to this relation. For ideal fluid, generally speaking, there is no universal rest frame where one could apply this relation. However, for the ideal fluid the symmetry group includes diffeomorphism and charge conservation is obeyed separately within each comoving fluid element (for an enlightening discussion of conservation laws for ideal fluids see [16]).

\subsection{Thermal vortical effect}

As is noted in Ref. [15] consideration of fluid conservation laws in external gravitational field allows to derive novel thermal chiral effects. One simply replaces the acceleration (in the appropriate units system) induced by the gravitational field by

$$
\vec{a}_{g r}=-\frac{\vec{\nabla} T}{T},
$$

as first proposed by Luttinger [14]. 
Following this suggestion we get for divergence of the fluid-helicity current:

$$
\partial_{\alpha} \epsilon^{\alpha \beta \gamma \delta}\left(\mu u^{\beta}\right) \partial^{\gamma}\left(\mu u^{\delta}\right)=-(\text { const }) \frac{\mu^{2}}{T} \vec{\Omega} \cdot \vec{\nabla} T
$$

This equation is very similar to that obtained in [15] for the same type of helical motion, with acceleration $\vec{a}$ being parallel to the angular velocity $\vec{\Omega}$, see Eq. (12). There is an important difference as well, however. Namely, in Ref. [15] the r.h.s. of Eq. (14) stands for the divergence of the total axial current, see (1). Let us remind the reader that the two terms in Eq. (1) for the axial current look diffierent mcroscopically. Namely, the standard hydrodynamic current refers to the flow of elementary chiral coinstituents while the fluid-helicity current describes macroscopic helical motion. Thus, the result of Ref. [15] allows for interpretation that it is the elementary constituents of certain chirality flowing along the vector $\vec{\nabla} T$ that are responsible for a nonvanishing divergence of $j_{\alpha}^{5}$. While in our case, see Eq. (14), there is no hint on mixing of the microcopic and macroscopic degrees of freedom, for further details and references see [17].

It is worth mentioning that the original proposal of Luttinger [14] referred to hydrodynamic motion in resistive media. In other words, there is flow of fluid along the gradient of the gravitational potential $\vec{\nabla} \phi_{g r}$ but with a constant velocity, without acceleration, $\vec{a}_{\text {fluid }}=0$. On the other hand, substitution (13) proposed in Ref. [15] refers to the dissipation-free motion, and is valid in high-frequency limit. Validity of this approximation is to be justified in each particular case.

\subsection{Emergent gravity}

Turn back to consideration of motion in flat space. For ideal fluid in equilibrium, the fluid helicity current, see Eq. (12) is conserved. However, for viscous fluids there is no such conservation law $[12,13]$. Indeed, for a viscous fluid the equilibrium configuration in most cases is nothing else but the whole of the fluid being at rest in a particular frame so that the helical charge vanishes.

It is speculated in [10] that at short times the effect of non-conservation of helicity can be described in terms of effective gravity. In more detail, let us start with motion of ideal fluid and then switch on non-vanishing viscosity. At short times, there appear acceleration which is proportional to the viscosity and we get a non-vanishing r.h.s. of Eq, (12). This acceleration is fixed in terms of the initial conditions of the motion of the ideal fluid. Furthermore, one could introduce effective gravitational field which reproduces this acceleration. In other words, the emergent gravitational field is fixed in terms of the motion of dissipative fluid. In this approximation, the conservation of the fluid helicity holds in terms of the respective covariant derivatives, see discussion above.

This qualitative picture does not contradict much more systematic general studies of theory of dissipative fluids. Ref. [18], e.g., introduces both an effective metric tensor, defined in terms of the motion of the fluid itself, and supersymmetry, responsible for the dissipation.

\section{Ultraviolet vs infrared sensitivity of the chiral effects.}

\subsection{Pion superfluidity}

In this section we review a simple toy model of chiral effects. One starts with pionic medium in the presence of chemical potentials, $\mu_{3, V}, \mu_{3, A}$ violating isotopic symmetry. The corresponding Lagrangian in terms of the quark fields is given by:

$$
\delta L=\mu_{3, V} \bar{q} \gamma_{0}\left(\tau_{3} / 2\right) q+\mu_{3, A} \bar{q} \gamma_{0} \gamma_{5}\left(\tau_{3} / 2\right) q,
$$


where $q$ are isotopic doublets, quark fields, $\gamma_{0}, \gamma_{5}$ are Dirac matrices, $\left(\tau^{3} / 2\right)$ is the generator of rotations around the third axis in the isotopic-spin space. It is well known that in the low-energy domain the model (15) exhibits pionic superfluidity. Although the derivation of this amusing result is absolutely straightforward, because of the space consideration we only sketch it. Details and further references can be found in $[19,20]$. Furthermore, switching on external magnetic fields and/or rotation one can derive the chiral effects which is our central point in this section. In presentation we follow [21, 22] where further details and references can be found.

The proof of superfluidity is given in terms of effective Lagrangians describing interaction of light degrees of freedom, or pions. Goldstone fields, or pions represent phases of a unitary matrix $U$ :

$$
U=\exp \left(i \tau^{a} \pi^{a} / f_{\pi}\right),
$$

where $f_{\pi}$ is a constant, $\tau^{a}(a=1,2,3)$ are Pauli matrices, $\pi^{a}$ are pion fields. The leading term in the effective Lagrangian has two derivatives and corresponds to the kinetic energy of the pions. To incorporate the effect of the chemical potentials one replaces ordinary derivatives by the covariant ones [19]:

$$
L_{\text {chiral }}=\frac{f_{\pi}^{2}}{4}\left(D_{\mu} U D^{\mu} U^{\dagger}\right)
$$

where the covariant derivatives are defined as:

$$
D_{\mu} U=\partial_{\mu} U-i \delta_{\mu 0}\left(\hat{\mu}_{3, L} U-U \hat{\mu}_{3, R}\right),
$$

and $\hat{\mu}_{3, L}, \hat{\mu}_{3, R} \equiv \mu_{3, L} \sigma_{3}, \mu_{3, R} \sigma_{3}$. The chemical potentials $\mu_{3, L}, \mu_{3, R}$ are related to $\mu_{V, 3}, \mu_{A, 3}$ introduced in Eq. (15) in an obvious way.

In fact, the cases $\left(\mu_{3, V} \neq 0, \mu_{3, A}=0\right)$ and $\left(\mu_{3, V}=0, \mu_{3, A} \neq 0\right)$ in the limit of exact chiral symmetry can be reduced to each other by a change in notations. The point is explained in detail in Ref. [20], We will consider $\mu_{3, A} \neq 0, \mu_{3, V}=0$. Then the potential energy $V_{\text {chiral }}$ corresponding to the Lagrangian (17) is given by :

$$
V_{\text {chiral }}=-\frac{f_{\pi}^{2}}{8} \mu_{3, A}^{2}\left(\operatorname{Tr}\left(U \sigma_{3} U^{\dagger} \sigma_{3}\right)+2 \operatorname{Tr} I\right)
$$

The minimum of the potential energy is reached on matrices $U_{\min }$ where

$$
U_{\min }=I \cos \phi+\sigma_{3} \sin \phi .
$$

The independence of the energy (19) on the angle $\phi$ implies presence of a Goldstone boson. The superfluid solution is associated with the phase growing with time:

$$
U_{\text {solution }}=\exp \left(i \sigma_{3} \mu_{3, A} \cdot t\right),
$$

In other words, the field $\pi_{0}$, defined in (16) looks as,

$$
\frac{\pi_{0}}{f_{\pi}}=\mu \cdot t+\varphi\left(x_{i}\right)
$$

where the function $\varphi\left(x_{i}\right)$ satisfies the equation $\Delta \varphi\left(x_{i}\right)=0$. The $3 \mathrm{~d}$ massless field $\varphi$ signifies superfluidity.

Differentiating the potential energy with respect to $\mu_{3, A}$ one gets the density of pions in the ground state:

$$
n_{5}=f_{\pi}^{2} \mu_{3,5} .
$$

A similar equation holds if one starts with $\mu_{3, V} \neq 0, \mu_{3, A}=0$.

As we argue in the next subsection, knowing Eq. (23) allows for a quick derivation of the chiral magnetic effect. 


\subsection{Chiral effects in superfluid as radiative corrections}

In this subsection we review theory of the chiral magnetic effect treated as a radiative correction to the relation (23). One first rewrites (23) as an expression for the hydrodynamic axial current:

$$
j_{\alpha}^{5}=f_{\pi} \partial_{\alpha} \pi^{0}=n_{5} u_{\alpha},
$$

where $n_{5}$ is the density of $\pi^{0}$-mesons. And then one accounts for the interaction of $\pi^{0}$ with external electromagnetic fields, following the standard rules of quantum field theory. Similar approach was exploited in, e.g., [23-25]. We follow closely [21, 22] and argue that in the hydrodynamic approximation evaluation of the chiral magnetic effect is factorized into calculation of the density (23) and of the amplitude of interaction of $\pi^{0}$-meson with external electromagnetic fields.

Decay of $\pi^{0}$ into two photons is governed by an effective vertex:

$$
\delta L=f_{\pi^{0} \rightarrow 2 \gamma} \pi^{0} \epsilon^{\alpha \beta \gamma \delta} \partial_{\alpha} A_{\beta} \partial_{\gamma} A_{\delta},
$$

where $f_{\pi^{0} \rightarrow 2 \gamma}$ is a constant, $\pi^{0}$ is the field of $\pi^{0}$-meson. Note that Eq. (25) incorporates in fact the chiral anomaly. Indeed, if there were no anomaly the vertex (25) would have vanished in the limit of vanishing 4-momentum of the pion and $f_{\pi^{0} \rightarrow 2 \gamma}$ could not be treated as a constant. By varying (25) with respect to the potential $A_{\alpha}$ one can generate an effective electromagnetic current $j_{\alpha}^{e l}$. Moreover, it is useful first to integrate (25) by parts. As a result,

$$
j_{\alpha}^{e l}=f_{\pi^{0} \rightarrow 2 \gamma} \epsilon_{\alpha \beta \gamma \delta}\left(\partial^{\beta} \pi^{0}\right) F^{\gamma \delta},
$$

where $F^{\gamma \delta}$ is the electromagnetic field strength tensor. Note that the current (26) was introduced first in Refs. [26, 27].

To make contact with hydrodynamics (of superfluid) we need to only substitute (24) to (1). It is readily seen that we do reproduce the chiral magnetic effect since in case we consider the constant $C_{\text {anom }}$ entering Eq (1) is given by

$$
C_{\text {anom }}\left(4 \pi \alpha_{e l}\right)=f_{\pi} \cdot f_{\pi^{0} \rightarrow 2 \gamma} .
$$

And this equation completes the derivation of the chiral magnetic moment in the model considered.

Now, that we have an explicit calculation of a chiral effect we can come back to questions mentioned in the Introduction. First, the mystery of dissipation-free transport is resolved now through the observation that the chiral magnetic effect corresponds to a polynomial in the effective action. Thus, there is no actual "transport" behind this effect. (Note, however, that the displacement current that we have in our model can be transformed into ordinary current on a subspace of lower dimension, see in particular [27].)

Another application of the technique outlined above is the evaluation of dependence of the chiral separation effect on pion mass. The chiral separation effect is associated with $\mu_{3, V} \neq 0$. Non-vanishing pion mass can consistently be incorporated in this case and $m_{\pi} \neq 0$ modifies in a well-defined way the density (23) [19]. Basing on considerations outlined in the current subsection one immediately concludes that the density of the pions in the ground state is the only source of the "fast" dependence of the chiral separation effect on the infrared-sensitive physics. For further details see [22].

\section{Chiral vortical effect}

\subsection{Duality between chiral vortical effect and spin of vortices}

There is another aspect of duality which is made manifested within field-theoretic approch. Consider next the chiral vortical current, see second line of Eq. (1). At first sight it looks as a next term in 
the derivative expansion. Thus, one could expect that specific hydrodynamic excitations, like sound waves, are responsible for the dynamics of the chiral vortical effect. Within the model we consider, however, the chiral votical effect can also be derived as a radiative correction to the supercurrent associated with short distances [21].

The reason is that the chiral vortical effect is saturated by the contribution of cores of vortices, not by long-wave hydrodynamic excitations, as we explain now in more detail. The starting point is the well known obsevation that rotation cannot be transferred directly to a superfluid. Indeed, Eq. (24) demonstrates that the velocity of the supecurrent $\vec{v} \sim \vec{\nabla} \varphi$ and $\vec{\nabla} \times \vec{v} \equiv 0$. is $\vec{j} \sim \vec{\nabla} \varphi$ and $\vec{\nabla} \times \vec{j}=0$ However, rotation is still possible if one allows for singularities, or vortices. Near the singularity,

$$
\frac{\pi^{0}}{f_{\pi}}=\mu_{5} \cdot t+\kappa \theta,
$$

where $\theta$ is the polar angle and $\kappa$ is integer. As a result,

$$
\left(\partial_{x} \partial_{y}-\partial_{y} \partial_{x}\right) \theta=2 \pi \delta(x, y)
$$

The condition that $\kappa$ is integer follows from the quantum nature of superfluidity. Namely, the phase of the wave function is to be single-valued and, therefore,

$$
\mu_{5} \oint v_{i} d x^{i}=2 \pi \kappa, \kappa=1,2 \ldots
$$

In applications, $\kappa=1$ in most cases.

Note that in the hydrodynamic approximation size of the core of the vortex shrinks to zero. Thus, it is commonly said that (30) determines spin of vortices. The spin of vortices defined in this way is proportional to the Planck constant, as it should be. Spin of the vortex is to be distinguished from the total angular momentum associated with a particular vortex. The latter can be estimated as

$$
\mathcal{L}=\int_{0}^{d_{\text {vortex }}} d r \cdot(\text { mass }) \cdot r \cdot v_{\phi} \sim d_{\text {vortex }}^{2}
$$

where $d_{v o r t e x}$ stands for the distance to the next vortex. This angular momentum is not quantized.

The total number of vortices, $n_{\text {vortex }}$ is determined by the condition that the total angular momentum of a bucket with supefluid rotated with angular velocity $\vec{\omega}$ is (approximately) the same as of a solid body of the same shape as the bucket, see, e.g., [28]. As a result, see, e.g., [29] and references therein:

$$
n_{\text {vortex }}=\frac{\mu_{5}}{\pi} \int_{A} d^{2} x\left|\omega_{z}\right|
$$

Averaging over vortices locally allows then to introduce a quasi-continuum picture.

So far we used hydrodynamics to identify the chiral vortical effect with spin of vortices. Field theoretically, we can evaluate the same effect as a radiative correction to the superfluidity itself. The logic is the same as used above in case of the chiral magnetic effect. In this way, one gets [21]:

$$
\delta j_{\alpha}^{5}=\frac{1}{4 \pi^{2} f_{\pi}^{2}} \epsilon_{\alpha \beta \gamma \delta}\left(\partial^{\beta} \pi^{0}\right)\left(\partial^{\gamma} \pi^{0} \partial^{\delta} \pi^{0}\right) .
$$

Moreover, the field $\pi^{0}$ in the vicinity of the axis of a vortex is given by Eq. (28). Substituting (28) into (33) we get a field theoretic expression for the chiral vortical effect which fits perfectly well the hydrodynamic derivation of it, for further details and references see [21]. 


\subsection{Chiral anomaly as "anomalous spin" relation}

For normalization, consider first heavy, i.e. non-relativistic, fermions with spin $S=1 / 2$ at temperature $T=0$. In the rotating frame spins of the particles are aligned with the vector of angular velocity $\vec{\Omega}$. Density of the spin is then:

$$
<\vec{S}>=\rho \frac{1}{2} \vec{n}_{\Omega}
$$

where $\vec{n}_{\Omega}$ is the unit vector in the direction of $\vec{\Omega}, \rho$ is the density of fermions, and the Planck constant $\hbar$ is put to unit.

The chiral vortical effect was evaluated first by Vilenkin [30]. In case of massless fermions:

$$
\vec{j}_{5}=\frac{\mu^{2}}{2 \pi^{2}} \vec{\Omega}
$$

Exploring this duality between (totally antisymmetric) fermion spin density and axial current one can interpret (35), at least formally, by saying that effectively the particles now look like fermions at rest with an induced spin

$$
\vec{S}=\vec{\Omega} \frac{1}{|\mu| 2 \pi^{2}}
$$

so that integration over the Fermi sphere produces the spin density (35). Note that the r.h.s. is in fact proportional to the Planck constant since it is associated with a loop graph (which is similar to the standard triangle anomalous graph, with the substitution (5) for the vertices).

Note that the relation between axial current and spin density tensor $S^{\mu, \nu \lambda}$ for Dirac field is due to the fact that it is totally antisymmetric and dual to the axial current.

$$
S^{\mu, \nu \lambda}=\frac{1}{2} \epsilon^{\mu \nu \lambda \alpha} J_{5 \alpha}
$$

resulting in the relation for the space components of axial current and spin density

$$
\vec{s}=\vec{j}_{5}
$$

The expression for spin per particle $\vec{S} \sim \vec{\Omega} / \mu$ compartible with (36) may be obtained by assuming that particle occupy a Fermi sphere of radius $\mu$ with the density fixed by uncertainty relation. The growth of baryon polarization with chemical potential [31] compatible with experimental data may signal that this density is in fact not reached.

Relation of the anomaly to a kind of chiral-invariant magnetic or spin moment has been studied in a number of papers, see, e.g., [32] and references therein. Concentrate for a moment on case of magnetic moment of a charged fermion. If one starts from a massive fermion, its magnetic moment is inverse proportional to its mass. However, the use of such a description assumes that the momentum transfer from magnetic field, $|\vec{q}|$ is much smaller than mass, $|\vec{q}| \ll m$. If one sends the mass to zero first, to approach the chiral limit, then the magnetic moment does not survive since the magnetic-type coupling violates chirality conservation. However, if one includes the effect of the anomaly, or Berry phase [32] then there arises coupling of the massless fermion to magnetic field, as if the fermion has chiral-invariant magnetic moment directed along the momentum and inverse proportional to its energy.

We expect that in a rotating coordinate system the total angular momentum $\overrightarrow{\mathcal{J}}$ is aligned with the vector of angular velocity $\vec{\Omega}$ :

$$
\overrightarrow{\mathcal{J}}=\overrightarrow{\mathcal{L}}+\overrightarrow{\mathcal{S}} \sim \vec{\Omega} / \mid \vec{\Omega}
$$


where $\overrightarrow{\mathcal{L}}$ and $\overrightarrow{\mathcal{S}}$ are the total orbital and spin angular momenta, respectively. In case of the pion superfluidity (and for a certain range of $|\vec{\Omega}|$ ) the alignment of $\overrightarrow{\mathcal{L}}$ to $\vec{\Omega}$ was demonstrated long time ago, see textbooks [28] and a brief discussion above. The total-spin part in this model is represented by the spin of the cores of the vortices, see for discussion above. Moreover, the spin angular momentum represents a quantum correction, as follows from the quantization condition (30). What is revealed by the consideration of the chiral vortical effect, see [21,29] and discussion above, is that the spin part $\overrightarrow{\mathcal{S}}$ is dual to the anomalous triangle graph. It is this demonstration of the duality between the triangle graph and spin of the vortices that makes definite the notion of the "anomalous spin" which we are introducing here.

\subsection{Field theoretic duality between IR and UV degrees of freedom}

Field theory allows to reduce the chiral vortical effect to the matrix element (33). One can estimate this matrix element within effective theory determining couplings of pions to heavy degrees of freedom, say, hyperons . [21]. Actually, this matrix element is saturated by contribution of heavy particles and, as a result, the spin of vortices can be related to the spin of hyperons. Note that hyperons are not originally included into the effective theory (17). However, the UV completion of the effective theory turns necessary to evaluate the matrix element (33). As a result, the chiral vortical effect, evaluated originally for massless fermions, gets related to average value of spin of heavy hyperons [21] ${ }^{1}$.

The construction might seem too far fetching to be true. It is amusing therefore to note that a similar connection between UV and IR degrees of freedom apparently holds in case of superfluid ${ }^{3} \mathrm{He}-\mathrm{A}[33,34]$. The effective theory contains massless fermions which are collective excitations while the UV degrees of freedom are provided by the atoms of ${ }^{3} \mathrm{He}$. One can demonstrate explicitly that the density of angular momentum can be expressed both in terms of the IR and UV (collective or corresponding to atoms) degrees of freedom. The system is nonrelativistic and all the calculations are explicit and under control.

\subsection{Acknowlegments}

We are thankful to P.G. Mitkin, A.V. Sadofyev, A. V. Vasiliev for useful discussions.

The work was supported by RFBR grant 17-02-01108.

\section{References}

[1] D.T. Son and P. Surowka, Phys. Rev. Lett. 103, 191601 (2009), 0906.5044.

[2] D. E. Kharzeev, K. Landsteiner, A. Schmitt, and H.-U. Yee, "Strongly Interacting Matter in Magnetic Field, Lect. Notes Phys. 871, 1 (2013).

[3] D. E. Kharzeev and Ho-Ung Yee, Phys.Rev. D84, 045025 (2011), 1105.6360.

[4] A. Avdoshkin, V.P. Kirilin, A.V. Sadofyev, and V.I. Zakharov, Phys. Lett. B 7551 (2016), 1402.3587.

[5] N. Yamamoto, Phys. Rev. D93 065017 (2016), 1511.00933.

[6] A.N. Redlich, Phys. Rev. Lett. 5218 (1984).

[7] Yu. Akamatsu and N. Yamamoto, Phys. Rev. Lett. 111052002 (2013), 1302.2125.

[8] Z.V. Khaidukov, V.P. Kirilin, A.V. Sadofyev, and V.I. Zakharov, “On Magnetostatics of Chiral Media", 1307.0138.

\footnotetext{
${ }^{1}$ The dissipation makes the link with other QCD approaches to polarization where absorptive phases are necessary.
} 
[9] A.V. Sadofyev, V.I. Shevchenko, and V.I. Zakharov, Phys. Rev. D83 105025 (2011), 1012.1958.

[10] V.I. Zakharov, "Notes on conservation laws in chiral hydrodynamics", 1611.09113.

[11] G. Prokhorov and O. Teryaev, arXiv:1707.02491 [hep-th].

[12] H.K. Moffatt, J. Fluid Mech. 35, 117 (1969).

[13] J. D. Bekenstein, Astrophys. Journ. 319207 (1987).

[14] J. M. Luttinger, Phys. Rev. A135 1505 (1964).

[15] G. Basar, D. E. Kharzeev, and I. Zahed, Phys. Rev. Lett. 111161601 (2013), 1307.2234.

[16] S. Dubovsky, Lam Hui, A. Nicolis, Dam Thanh Son, Phys.Rev. D85, 085029 (2012), 1107.0731.

[17] P.V. Minkin, A.V. Vasiliev, V.I. Zakharov, in preparation.

[18] M. Crossley, P. Glorioso, and H. Liu, JHEP, 1709, 095 (2017). 1511.03646.

[19] D.T. Son and M. A. Stephanov, Phys.Rev.Lett. 86592 (2001), e-Print: hep-ph/0005225.

[20] O. Aharony, K. Peeters, J. Sonnenschein, and M. Zamaklar, JHEP, 0802 (2008) 071, 0709.3948.

[21] O. V. Teryaev and V. I. Zakharov, Phys. Rev. D96, 096023 (2017), 1705.01650.

[22] A. Avdoshkin, A.V. Sadofyev, and V.I. Zakharov, "IR properties of chiral effects in pionic matter", 1712.01256.

[23] D.T. Son and M.A. Stephanov, Phys.Rev. D77, 014021 (2008), 0710.108.

[24] M. Lublinsky and I. Zahed, Phys.Lett. B684, 119 (2010), 0910.1373.

[25] T. Kalaydzhyan, Phys.Rev. D89 105012 (2014), 1403.1256.

[26] J. Goldstone and F. Wilczek, Phys. Rev. Lett. 47, 986 (1981).

[27] C. G. Callan, Jr. and J. A. Harvey, Nucl. Phys. B250 427 (1985).

[28] L.D. Landau and E.M. Lifshitz, “Fluid Mechanics”, Pergamon Press (1959).

[29] V.P. Kirilin, A.V. Sadofyev, and V.I. Zakharov, Phys.Rev. D86, 025021 (2012), 1203.6312.

[30] A. Vilenkin, Phys.Rev. D20, 1807 (1979).

[31] A. Sorin and O. Teryaev, Phys. Rev. C 95, no. 1, 011902 (2017), 1606.08398.

[32] D. T. Son and N. Yamamoto, Phys.Rev. D87, 085016 (2013), 1210.8158.

[33] T. Kita, J. Phys. Soc. Jap 67, 216 (1998).

[34] G. E. Volovik and A. Vilenkin, Phys.Rev. D62, 025014 (2000), hep-ph/9905460. 